\title{
Design and Flow Field Analysis of Self-excited Oscillation Novel Electrostatic Spraying Blade
}

\author{
Zhaohui Wang $^{\mathrm{a}}$, Sheng Zhong ${ }^{\mathrm{b}}$, Faping Wang $^{\mathrm{c}}$ and Zhengjiang Pan ${ }^{\mathrm{d}}$
}

College of Mechanical Automation, Wuhan University of Science and Technology,

Wuhan 430081, China;

azhwang@wust.edu.cn, bwzhuiboy@sohu.com, '986144342@qq.com, d1018927544@qq.com

Keywords: Self-excited oscillation, spraying blade design, flow field distribution

\begin{abstract}
A novel self-excited oscillation spraying blade has been designed and the geometric model of the conical surface and rectangular surface self-excited oscillation spraying blade have been established. The flow field distribution of novel spraying blade has been obtained by finite element analysis, simulation results show that: the velocity is reverse close to both sides of the self-excited oscillation chamber, and the recirculation zone has been produced. From the lower spraying nozzle to the exit area of the oscillation chamber, the fluid speed is longitudinal symmetric distributed while the maximum speed appears at the junction of the lower spraying nozzle and collision wall, and the energy no loss after fluid through the oscillation chamber. Velocity distribution at the edge of conical surface self-excited oscillation spraying blade is more stable and uniform than rectangular surface self-excited oscillation spraying blade, the spraying area of the novel spraying blade can be increased, and the spraying atomization effect is greatly improved.
\end{abstract}

\section{Introduction}

Electrostatic spraying is a new technology widely used today by using an electrostatic field to improve the atomization effect of the oil, it makes oil through the spraying equipment being charged by different methods and form charged droplets group, and it not only has obvious effects on making the droplets uniform and refine, improving deposition, uniformity and adsorption of droplets on the target surface, saving energy, but also protecting the environment. However, overall, the existing electrostatic spraying still exists not only the problems including the oil atomization average particle size is larger, strip with uneven surface coating and uncontrolled oil quantity, but also the excessive oil consumption, the serious environmental pollution, which can not meet the requirements for high-quality strip surface spraying [1]. Currently one of the hot spots of spraying technology research is the self-excited oscillation pulsed jet generated by the principle of fluid dynamics, fluid resonance, fluid elastic, and underwater acoustics. The advantage of the self-excited oscillation is that it does not need external exciting source, which can generate a pulse jet using structure change of chamber with larger pressure characteristics and cavitation [2].

The self-excited oscillation pulsed effect has been widely used in mining, cutting and cleaning and the process by using fluid self-excited oscillation effects will have an important influence on electrostatic spraying on the strip surface [3]. In this paper, a novel electrostatic spraying blade structure has been designed, and blade geometry has been established based on jet self-oscillating effect. On the basis of the control equation, a numerical simulation of the internal flow field of novel spaying blade was carried out by using finite element analysis. According to the simulatuion results, the spraying blade chamber internal velocity field, velocity vector field, the trace of the fluid particle and the blade edge droplet exit velocity distribution uniformity were analysed, and compared with the correlative analysis of the original blade in order to determine the rationality of the novel spraying blade design, so as to improve the effect of oil spray atomization. This study will provide a theoretical basis for the development of a new generation of electrostatic spraying blade. 


\section{Novel model}

The structure of self-excited oscillation spraying blade. The design structure diagram of novel spraying blade is shown in Figure 1. In the oscillation chamber of spraying blade, when a high-speed oil flows through the top spraying gap to the downstream into the asymmetric oscillation chamber, high-speed jet and surrounding stationary gas occurs turbulent mixing, results in energy exchange and forms unstable shear layer. Since the jet velocity is larger, and the shear layer is unstable, the fluid around shear layer is entrained to form axial symmetry vortex. Vorticity disturbance of a certain frequency range in the oscillation chamber interior is amplified by the selective amplification of unstable shear layer, forms a large scale vortex structure and a series of discrete vortex, pressure disturbance waves are sprayed colliding with the downstream collision wall and the upstream of the nozzle (propagation at the speed of sound to the upstream). At this time, the new vorticity disturbance is induced in the sensitive area of the upstream of the nozzle again, the cycle of vorticity disturbance-amplification-production of the new vorticity fluctuation is formed and lead to the periodical change of the fluid impedance in blade oscillation chamber. The modulation process of "completely block," "partially block" and "do not block" to the jet are completed. The process is repeated to form a strong self-excited oscillation effect in the chamber. Finally, the spraying oil through the bottom spraying oil gap and the oil export can be fully atomized [4]. The design of the novel spraying blade increases the self-excited oscillation chamber based on the original blade structure, and makes the continuous spraying oil become into the pulsating spraying oil inside the spraying blade. The pressure peaks of the oil export is higher than the inlet pressure of $15 \%-30 \%$, and makes the export oil obtain the more uniform atomizing effect, so as to improve oiled quality and reduce the fuel consumption.
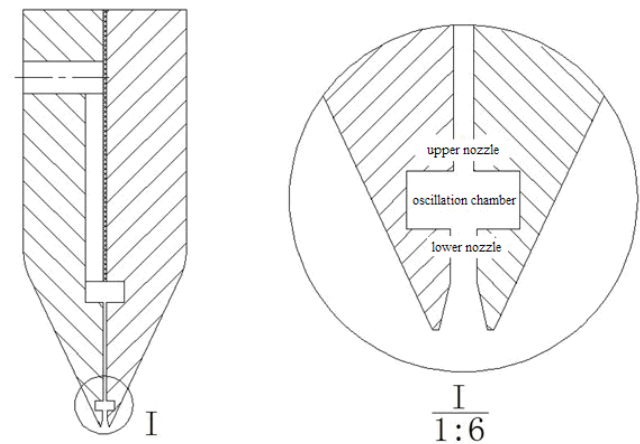

Fig 1. Structure diagram of the novel spraying blade

The geometric model of spraying blade. Through the analysis of the main factors affecting the effect of jet atomization of the self-excited oscillation, the key of the novel blade structure design lies in the design of the self-excited oscillation chamber. The main parameters of the chamber structure affecting self-excited oscillation are the upstream and downstream jet nozzle diameter, the collision wall shape, diameter and the shape of the chamber. Based on the existing electrostatic spraying blade structure, this paper selects the novel spraying blade structure of the conical surface oscillation chamber and the rectangular surface oscillation chamber as the research objects, the oscillation chamber structure and main design parameters as shown in Figure 2.

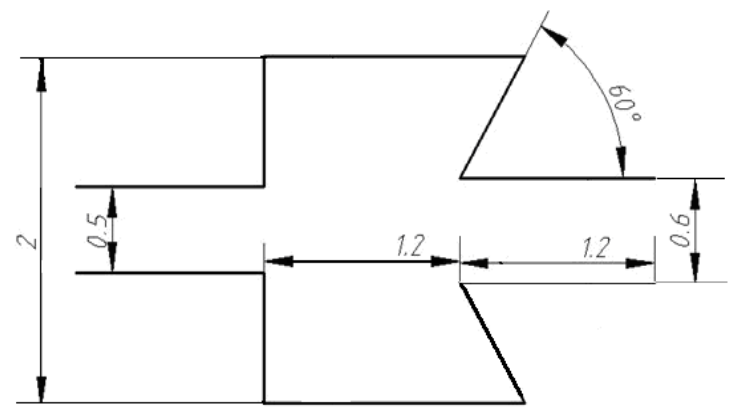

(a) Conical surface

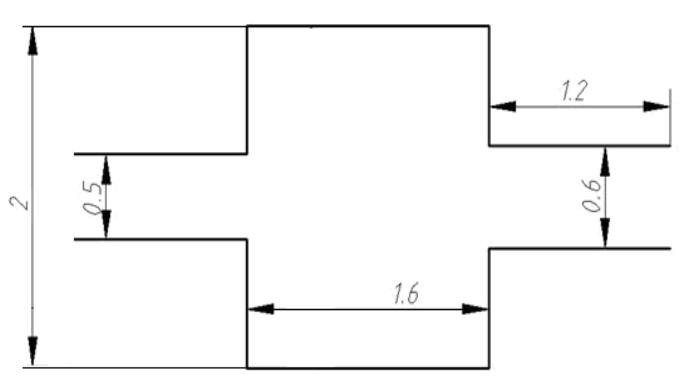

(b) Rectangular surface

Fig 2. Structure diagram of different self-excited oscillation chambers of novel spraying blade 


\section{Results and analysis}

Flow field distribution in the self-excited oscillation chamber. The flow field distribution in the conical surface of self-excited oscillation electrostatic spraying blade as shown in Figure 3, it can be seen, the velocity is reverse close to both sides of the self-excited oscillation chamber, and the recirculation zone has been produced. When the fluid passes through the mutant channels in the oscillation chamber, it can not turn back like the side wall suddenly, inevitable occurring the phenomenon that the mainstream is divorced from the boundary at the place where the side wall is mutant and forming the vortex area. From the lower spraying nozzle to the exit area of the oscillation chamber, the fluid speed is longitudinal symmetric distributed. The maximum speed of the fluid within the area of the calculation of the blade is $3.08 \mathrm{~m} / \mathrm{s}$, the maximum speed appears at the junction of the lower spraying nozzle and collision wall, the minimum speed is $0.00293 \mathrm{~m} / \mathrm{s}$. After the oil passes through the self-excited oscillation chamber, especially after by passing the oscillation chamber collision wall, the velocity will increases from $2.50 \mathrm{~m} / \mathrm{s}$ of the top nozzle to $3.08 \mathrm{~m} / \mathrm{s}$. This is concordant with the condition of "fluid oscillation through feedback, amplification, energy is gathered" theory among the self-excited oscillation theories [5]. The overall speed within the flow field area from the bottom nozzle to the chamber outlet keeps at about $2.25 \mathrm{~m} / \mathrm{s}$ and decreases slightly compared with the initialization velocity value set up of the top nozzle, the reducing amplitude is $0.25 \mathrm{~m} / \mathrm{s}$, it indicates that the energy does not loss after fluid through the oscillation chamber.

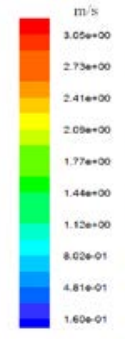

(a) Velocity contour

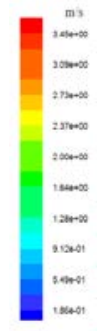

(b) Enlaregd velocity vector

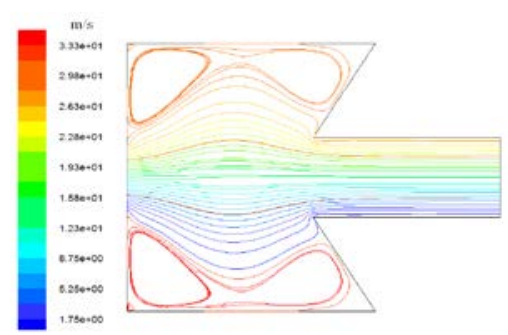

(c) Particle trajectories

Fig 3. Velocity distribution of the conical surface self-excited oscillation spraying blade

The distribution of the flow field in rectangular surface self-excited oscillation novel electrostatic spraying blade as shown in Figure 4, it can be seen, inside the oscillation chamber, the velocity is also reverse close to the both sides of the chamber, and the recirculation zone has been produced. From the lower spraying nozzle to the exit area of the oscillation chamber, the fluid speed is longitudinal symmetric distributed, the maximum speed of the oil in the blade calculation area is $2.80 \mathrm{~m} / \mathrm{s}$, the minimum speed is $0.0118 \mathrm{~m} / \mathrm{s}$, the maximum speed also appears at the junction of the lower spraying nozzle and collision wall. Due to the existence of the self-excited oscillation chamber, the velocity of the fluid will increases from $2.50 \mathrm{~m} / \mathrm{s}$ of the top nozzle to $2.80 \mathrm{~m} / \mathrm{s}$ after bypassing the collision wall.

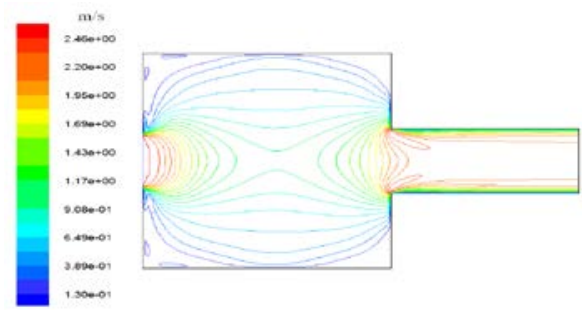

(a) Velocity contour

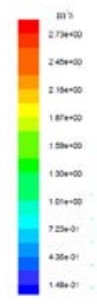

(b) Enlarged velocity vector

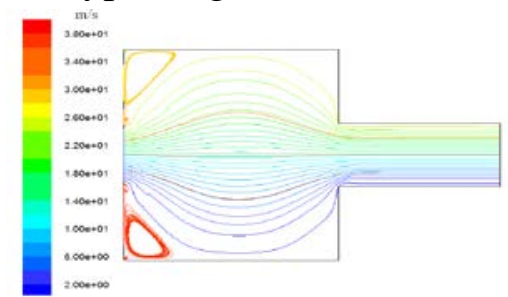

(c) Particle trajectories

Fig 4. Velocity distribution of the rectangular surface self-excited oscillation spraying blade The uniformity of droplet velocity at the edge of the blade. The distribution of the droplet velocity at the edge of the conical surface and rectangular surface self-oscillating spraying blade as shown in Figure 5, it can be seen, the droplet velocity at the exit in the two programs is longitudinal symmetric distributed. The maximum speed of the oil droplet at the outlet is approximately $2.20 \mathrm{~m} / \mathrm{s}$, the velocity value close to the chamber wall is approximately 0 , the maximum velocity value appears at the blade edge close to the axis of the location of the distribution in both sides. The droplet velocity distribution uniformity of the new blade is better than the original blade, the velocity distribution at the edge of the conical surface blade is more stable and uniform than the rectangular surface blade. The spraying 
area of the novel spraying blade can be increased, and the spraying atomization effect is greatly also improved.

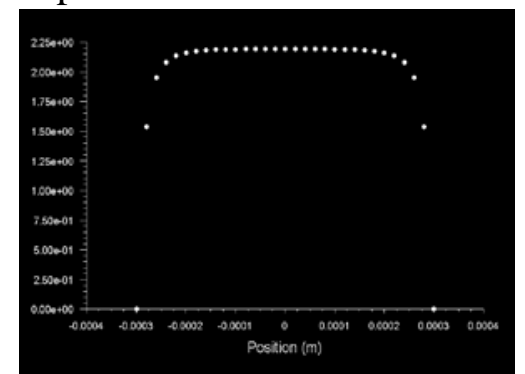

(a) Conical surface

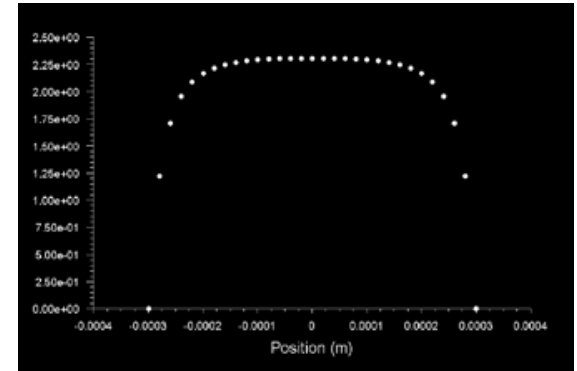

(b) Rectangular surface

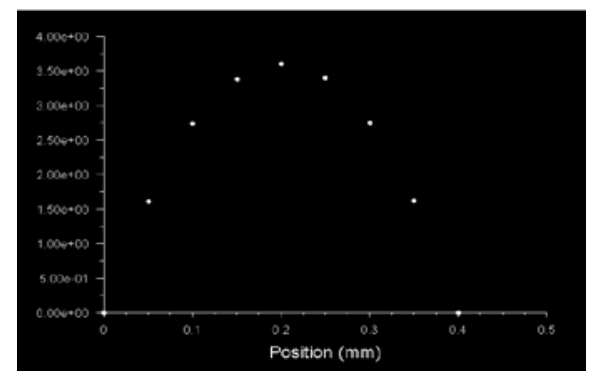

(c) The original blade

Fig 5. Droplet velocity distribution at the outlet of different types of novel spraying blade

\section{Summary}

The concept of self-excited oscillation electrostatic spraying has been put forward. The novel spraying blade structure has been designed, and the geometry model of the conical surface and rectangular surface self-excited oscillation spraying blade has been established. In the flow field distribution of the conical surface and rectangular surface self-excited oscillation spraying blade, the velocity is reverse close to both sides of the self-excited oscillation chamber and the recirculation region has been produced. From the lower spraying nozzle to the exit area of the oscillation chamber, the fluid speed is longitudinal symmetric distributed while the maximum speed appears at the junction of the lower spraying nozzle and collision wall. The droplet velocity at the blade edge decreases slightly compared with the set up initialization velocity value of the top nozzle, the fluid is no loss of energy through the self-excited oscillation chamber.

In the droplet velocity distribution of the self-excited oscillation spraying blade edge, the novel spraying blade has better oil atomization effect and better uniformity of the droplet. Velocity distribution at the edge of conical surface self-excited oscillation spraying blade is more stable and uniform than rectangular surface self-excited oscillation, the spraying area of the novel spraying blade can also be increased.

\section{Acknowledgements}

This project was supported by National Natural Science Foundation of China (51405352, 51376204).

\section{References}

[1] H. Yanada, T. Okamoto, D. K. Tran, Fundamental investigation of charge injection type of electrostatic oil filter, Journal of Electrostatics. 69 (2011) 180-188.

[2] Z. F. Liao, J. Li, D. S. Chen, Theory and experimental study of the self-excited oscillation pulsed jet nozzle, Chinese Journal of Mechanical Engineering. 16 (2003) 379-383.

[3] Y. Fei, R. Akira, Characteristics of particle velocity and concentration in a horizontal self-excited gas-solid two-phase pipe flow of using soft fins, International Journal of Multiphase Flow. 41 (2012) 68-76.

[4] Z. H. Wang, Q. J. Gao, Z. M. Xu, Design and numerical simulation of gas-liquid mixing novel spraying blade, Journal of Drainage and Irrigation Machinery Engineering. 31 (2013) 236-241.

[5] Z. H. Han, G. Y. Wang, Fundamental Fluid Mechanics. Beijing Institute of Technology Press, Beijing, 2012. 